\title{
La lactancia como eje integrador \\ de actividades en docencia, investigación y extensión
}

\author{
Hebe P. Rojo \\ heberojo@gmail.com
}

\section{Silvia Mirkin}

silviamirkin@gmail.com

\section{Silvina Aguirre}

silvinaaguirret@gmail.com

Docentes investigadoras de la Facultad de Medicina. Universidad Nacional de Tucumán, Argentina.

\author{
Andrea Giorgis \\ Médica residente del Hospital de \\ Pediatría Juan P. Garrahan de la \\ Ciudad Autónoma de Buenos Aires, \\ Argentina. \\ Voluntaria de proyectos de \\ extensión de la Universidad \\ Nacional de Tucumán. \\ andrea.giorgis.88@gmail.com
}

Integración de la docencia y la extensión /

Intervenciones

RECEPCIÓN: 24/06/16

ACEPTACIÓN FINAL: 10/10/16

\section{Resumen}

Promover la lactancia materna constituye una sencilla estrategia de prevención de la morbimortalidad infantil. Las decisiones y actitudes del equipo de salud condicionan los resultados de lactancia en una población. Por ello, capacitar a estudiantes de Medicina y motivarlos a participar en actividades diversas relacionadas con la lactancia constituye una estrategia de promoción a largo plazo. Se describen en este trabajo actividades encuadradas en la docencia, la extensión y la investigación que se realizan en la Facultad de Medicina de la Universidad Nacional de Tucumán tomando como eje a la lactancia. La docencia está representada por el Módulo Optativo Lactancia Materna, que se articula con el Voluntariado Universitario Apoyo a la Lactancia Materna y con el Proyecto de investigación sobre la misma temática. Se destaca la participación activa de los estudiantes y el valor de esta experiencia para su formación profesional y humana.

Palabras-clave

- Lactancia materna

- Integración

- Docencia

- Investigación

- Extensión

\section{Resumo}

Promover a amamentação constitui uma estratégia simples de prevenção da morbidade e mortalidade na infância. As desisões e atitudes da equipe de saúde condicionam os resultados da amamentação em uma população. Por isso, capacitar estudantes de medicina e motivá-los a participar em diversas atividades relacionadas com a amamentação constitui uma estatégia de promoção a longo prazo. Descrevem-se neste artigo atividades enquadradas na docência, na extensão e na pesquisa que se realizam na Faculdade de Medicina da Universidade Nacional de Tucuman, tomando como eixo a amamentação. A docência é representada pelo "Módulo Opcional de Amamentação", que se articula como o "Voluntariado Universitário Apoio à Amamentação" e com o projeto de pesquisa sobre o mesmo tema. Destaca-se a participacão ativa dos alunos e o valor dessa experiência para a sua formação profissional e humana.

Palavras-chave

- Lactância materna

- Integração

- Ensino

- Pesquisa

- Extensão 


\section{Introducción}

La lactancia materna beneficia la salud del niño y de la madre y fortalece el vínculo entre ambos. La leche materna posee todos los nutrientes necesarios para el bebé y compuestos biológicos que le brindan defensa contra numerosas infecciones.La lactancia se constituye así en un modo sencillo para prevenir la morbimortalidad infantil. La alimentación a pecho es la estrategia de intervención que mayor número anual de muertes de menores de un año puede evitar, muy por encima de otras intervenciones exitosas, como suministrar agua potable o implementar vacunación antitetánica (Jones et al., 2003). El inicio del amamantamiento después del parto puede evitar el $16 \%$ de las muertes neonatales si se lo inicia desde el primer día de vida, incrementándose al $22 \%$ si el inicio es durante la primera hora de vida (Edmond et al., 2006).

Actualmente las normas, tanto del Ministerio de Salud de la Nación Argentina, como las recomendaciones de la Academia Americana de Pediatría y de la Sociedad Argentina de Pediatría, se basan en el criterio de la Organización Mundial de la Salud (OMS) y el Fondo de las Naciones Unidas para la Infancia (Unicef) de promover la lactancia materna exclusiva hasta el sexto mes de vida y mantenerla con alimentación complementaria oportuna hasta los dos años o más.

A nivel mundial, el porcentaje de niños de 0 a 5 meses que fueron alimentados exclusivamente con leche materna en las pasadas 24 horas es del $37 \%$, según el informe de Unicef (2012) sobre el estado mundial de la infancia. En Argentina, los datos de la Encuesta Nacional de Nutrición y Salud (2007) muestran que el $95 \%$ de los niños inicia su alimentación mediante lactancia materna pero, a medida que transcurren los meses, esta práctica comienza a perderse, y es del $36 \%$ a los seis meses. Mientras que el informe del Ministerio de Salud denominado "Situación de la Lactancia Materna en Argentina" (2011) consigna que la Lactancia Materna Exclusiva a los seis meses es del $30 \%$. A pesar de que los beneficios de la lactancia se extienden al niño, su madre, la familia y la comunidad en su conjunto, estos datos muestran la necesidad de generar estrategias para fortalecer esta práctica. Un camino sería acercarse a las madres, escuchar sus dificultades y apoyarlas para sostener la lactancia; otro, trabajar en la formación de estudiantes que integrarán los equipos de salud. Las decisiones y actitudes del equipo de salud condicionan los resultados de lactancia de una población. Por ello, capacitar a los estudiantes de Medicina y comprometerlos en actividades de investigación y extensión relacionadas con la lactancia y sus beneficios es una estrategia de promoción a largo plazo.

El objetivo de este trabajo es describir las actividades realizadas en la Facultad de Medicina de la Universidad Nacional de Tucumán (UNT), Argentina, tomando como eje a la lactancia, encuadradas en la docencia, la extensión y la investigación, y destacando la participación de los estudiantes así como el valor para su formación profesional y humana y para la comunidad de la cual forman parte.

\section{La formación de estudiantes}

En el año 2001 se firmó un Acuerdo de Cooperación entre la Asociación de Facultades de Ciencias Médicas de la República Argentina (AFACIMERA) y Unicef con el objetivo de fortalecer la enseñanza de la lactancia materna en los primeros años de las carreras de Medicina del país. Para el desarrollo de la propuesta se capacitó a docentes del ciclo básico y se editó el Manual de Autoinstrucción Lactancia Materna. Bases científicas para la práctica clínica, para servir de guía (Waisman, 2003). La propuesta fue integrar contenidos de asignaturas básicas, como Anatomía, Bioquímica, Histología, Fisiología y Salud Pública, tomando como eje de contenidos a la lactancia (Galli et al., 2009). Diversas universidades argentinas, en sus carreras de Medicina, implementaron como materia electiva a la Lactancia Materna.

Tal es el caso de las Universidades Nacionales de Córdoba, Comahue, Nordeste y Tucumán. El Módulo Optativo Lactancia Materna destinado a estudiantes del ciclo básico de la carrera de médico de la Facultad de Medicina de la UNT se creó a fines del año 2005 y comenzó a dictarse formalmente en 2006; antes se había realizado un curso en calidad de prueba piloto.

Los objetivos generales definidos para el Módulo implementado en la UNT fueron:

- Capacitar a los estudiantes en los fundamentos científicos de las ventajas de la lactancia.

- Sensibilizarlos con la importancia de la temática.

- Fortalecer su compromiso con actividades de promoción en la comunidad.

- Despertar el interés en la investigación sobre el tema.

- Contribuir a formar profesionales que fomenten y favorezcan la lactancia en su ejercicio profesional futuro.

Los docentes a cargo del dictado pertenecen a la cátedra de Bioquímica, si bien en varias oportunidades han realizado su aporte docentes de otras cátedras, como Pediatría y también profesionales de instituciones de salud.

Las estrategias didácticas empleadas son variadas: clases teóricas expositivas, estudio independiente sobre la base de la lectura y actividades planteadas en el Módulo de autoinstrucción, encuentros en grupos pequeños, análisis de casos clínicos, dramatizaciones, trabajo práctico sobre medicamentos utilizando una página web y visita a madres en etapa de lactancia.

El cursado del Módulo se evalúa desde la perspectiva de los estudiantes participantes.

Al inicio y al final del cursado se distribuye a los estudiantes la misma encuesta, sobre conocimientos generales de lactancia, para valorar la superación de preconceptos erróneos. En la encuesta de final del cursado también se pregunta si recomendaría el cursado del Módulo a compañeros y se solicita consignar aspectos positivos y negativos del mismo. 
Los resultados de la encuesta de conocimiento, impartida al inicio del Módulo, se tomaron como evaluación diagnóstica para determinar los conocimientos previos de los estudiantes. La lactancia en muchos aspectos puede considerarse un saber de la vida diaria y dichos conocimientos bien podrían haberse obtenido de la familia, la escuela o los medios de comunicación masiva. Sin embargo, a pesar de ser un grupo de estudiantes que se proyecta como equipo de salud, se detecta la existencia de conceptos erróneos en muchos de ellos. Estos resultados fueron el punto de partida para fortalecer el proceso didáctico en torno a aquellos aspectos que presentaron un mayor desconocimiento, tales como el concepto de lactancia exclusiva o el momento óptimo para iniciar la lactancia. Comparando los resultados de la encuesta de conocimiento al inicio y al final del Módulo, para cada una de las diez preguntas se pudo demostrar un incremento del porcentaje de respuestas correctas finalizado el Módulo, lo que refleja la superación de preconceptos erróneos.

En cuanto a las opiniones de los estudiantes, el 100 \% expresó que recomendaría el cursado del Módulo a otros compañeros y destacó, sobre todo, la organización del mismo, la multiplicidad de actividades planteadas, la importancia de la temática y el compromiso de los docentes. Entre los aspectos negativos, uno recurrente fue el poco tiempo (se desarrolla en cuatro semanas) y lo numeroso del grupo de estudiantes (no existe un límite para el número de inscriptos pero sí un limitado grupo de docentes, aunque fue creciendo con los años).

El número de inscriptos y el de aprobados refleja el creciente entusiasmo de los estudiantes. A lo largo de nueve cursados se inscribió un total de 1152 estudiantes, de los cuales 924 lo aprobaron. El $93 \%$ de los que cumplieron los requisitos para rendir el examen final alcanzó calificaciones iguales o superiores a 8 , en escala de 1 a 10 . El número de inscriptos fue incrementándose con los años y superó los 180 en 2016. La valoración de los estudiantes sobre las diferentes estrategias empleadas permitió ir fortaleciendo algunas o modificando otras. Por ejemplo, se incrementó el uso de nuevas tecnologías mediante la puesta en funcionamiento de un aula virtual donde se incorporaron videos y el material de estudio obligatorio y complementario.

El aporte de los docentes al desarrollo del Módulo también fue importante. En un comienzo se acompañaba a los estudiantes a los centros asistenciales a realizar una visita a madres, con la colaboración de personal de dichos centros. Con el crecimiento del número de estudiantes, esta actividad se hizo casi imposible. La propuesta del equipo docente fue que cada alumno buscara una madre que estuviera amamantado, ya sea en su barrio, familia, o en un centro asistencial, para entrevistarla y completar una guía de observación de amamantamiento, previo consentimiento informado de la madre.

Dado que el equipo docente del Módulo participa también en cursos de capacitación a profesionales, contenidos requeridos por éstos fueron igualmente incorporados al Módulo, tal es el caso del tema "Medicamentos y Lactancia Materna".

\section{De las aulas a la comunidad}

Finalizado cada cursado del Módulo, un grupo de estudiantes sensibilizados con la temática y su importancia para la salud decidió continuar en una segunda etapa fuera de las aulas, inmersos en la comunidad.

Las actividades de extensión tienen lugar en el marco del Voluntariado Universitario denominado "Apoyo a la Lactancia Materna". El Voluntariado ha sido aprobado, en forma ininterrumpida, en las convocatorias del Programa de Voluntariado Universitario del Ministerio de Educación de la Nación realizadas desde el año 2006 a la fecha. Ha recibido una mención especial del Premio Presidencial Prácticas Educativas Solidarias en Educación Superior 2008 y otras distinciones.

La participación estudiantil en este proyecto de extensión varía entre 15 y 40 estudiantes por año. Parte del equipo docente del Módulo pertenece a la cátedra de Bioquímica y también participan docentes de la Escuela de Enfermería de la UNT, quienes además trabajan en hospitales de la capital y del interior de Tucumán. Esta composición del grupo facilitó mucho la articulación con dichos hospitales. La elección de los centros asistenciales en los cuales desarrollar la actividad de extensión está condicionada a la voluntad de los directivos de firmar las Actas Acuerdo y recibir a los estudiantes.

El "Voluntariado Apoyo a la Lactancia Materna" busca dar una respuesta a la problemática del abandono temprano de la lactancia. Mediante una estrategia conjunta entre estudiantes, docentes y actores institucionales, tiende a fortalecer la práctica de la lactancia materna entre las madres de diferentes zonas de la provincia de Tucumán. El propósito es concientizar a las madres sobre la importancia y los beneficios de la lactancia materna y fortalecer su decisión de amamantar. 


\author{
el Voluntariado Apoyo a la Lactancia \\ Materna busca dar una respuesta a la \\ problemática del abandono temprano \\ de la lactancia
}

Las principales destinatarias son embarazadas y puérperas que concurren a centros asistenciales de diversas regiones de Tucumán. Los espacios de acción incluyen hospitales situados tanto en la capital como en el interior, centros de Atención Primaria de Salud, centros de integración comunitaria, fundaciones y espacios no convencionales, como plazas, peatonal céntrica y exposiciones rurales.

Los ejes de promoción se desarrollan en tres instancias: el diálogo personal con una madre, talleres dirigidos a grupos de madres con ayuda de láminas o proyecciones y la distribución de folletos a la población en general. Las destinatarias del proyecto fueron alrededor de 400 madres por año.

Del trabajo conjunto entre voluntarios, docentes y actores de las instituciones de salud, surgieron los temas centrales, relativos a la lactancia, a desarrollar en las instancias de promoción. Los mismos fueron plasmados en láminas y organizados en un rotafolio para utilizarse en los talleres y también en folletos.

Para medir el impacto de las acciones de promoción, antes y después de los talleres se realizan sendas encuestas a las madres con preguntas generales de lactancia. También se les pregunta sobre su grado de satisfacción con los talleres protagonizados por los voluntarios. Transcurrido un período de 3 a 6 meses, se contacta telefónicamente a las madres para preguntarles sobre la lactancia de su hijo durante los primeros seis meses de edad. Los resultados de las encuestas a las madres mostraron un mayor porcentaje de respuestas correctas, a las preguntas formuladas luego del taller. En cuanto a la opinión de las madres sobre la actividad, el $72 \%$ de ellas calificó al taller como "Excelente" y el $18 \%$ como "Muy Bueno". El $91 \%$ de las madres expresó que el taller o la consejería les permitieron quitarse dudas sobre la lactancia. El 96 \% manifestó la voluntad de que su bebé reciba solo leche materna hasta el sexto mes de vida (datos correspondientes a 2013). Las llamadas telefónicas, realizadas más adelante, presentaron algunas dificultades. Solamente pudo contactarse a dos tercios del total de madres, ya que muchas probablemente habían cambiado de número telefónico o en otros casos respondía otra persona, o simplemente no tenían teléfono. Lo gratificante fue que un porcentaje elevado ( $77 \%$ de las madres localizadas telefónicamente, destinatarias de 2013) manifestó que alimentó a sus hijos con lactancia exclusiva hasta los seis meses. Algunas incluso relataron cómo apoyaron la lactancia de familiares o vecinas, lo que puso de manifiesto la multiplicación de las acciones del voluntariado.

En cuanto a las organizaciones, los centros de atención primaria y hospitales donde se llevan a cabo la mayoría de las acciones de promoción se vieron beneficiados por el apoyo de los voluntarios. Los estudiantes colaboraron anualmente en la organización de la Semana Mundial de la Lactancia Materna mediante el diseño y ejecución de juegos destinados a las madres, así como en la elaboración y distribución de folletos sobre la temática.

\section{Investigación}

La investigación se realiza en el área didáctica, en el laboratorio bioquímico y en la comunidad, en el marco de un Proyecto de investigación aprobado por el Consejo de investigaciones de la UNT (PIUNT) para el período 2014-2017. El mencionado Proyecto se titula: "Beneficios de la lactancia materna: fundamentos bioquímicos, enseñanza y promoción" y se desarrolla también en la cátedra de Bioquímica. Es continuación de otro proyecto aprobado en el año 2008 titulado "Estudio integrado de la lactancia materna: Bioquímica, Didáctica y Promoción”. Participa un número reducido de alumnos, actualmente dos. En el laboratorio se estudian propiedades bioquímicas de la leche humana tales como actividad antioxidante.

La investigación pedagógica aborda cada dictado del Módulo a través de los resultados de aprendizaje, la evaluación de las estrategias y la satisfacción de los estudiantes con el cursado. 
En la comunidad se evalúan los conocimientos de las madres sobre lactancia y la satisfacción con los talleres, como se mencionó anteriormente.

En cuanto a la formación de recursos humanos, se desarrolla en el marco institucional a través de becas y dirección de trabajos finales de estudiantes del último curso de la carrera de Médico. Por lo general se trata de estudiantes que aprobaron el Módulo, fueron voluntarios y en el último año decidieron abordar su trabajo final sobre una temática relativa a la lactancia Se dirigieron 11 becas cuyos temas de trabajo fueron sobre propiedades bioquímicas de la leche humana, y sus beneficiarios fueron en su mayoría estudiantes. Por otra parte, diez alumnos realizaron sus trabajos finales de la carrera de Médico sobre temáticas relacionados con la lactancia en el medio rural donde les tocó actuar durante la pasantía que realizan en el último curso.

\section{Conclusiones}

Estas experiencias, protagonizadas por alumnos y docentes de la Facultad de Medicina de la UNT junto a actores institucionales, se construyen desde variadas perspectivas que posibilitan distintas miradas de la lactancia y, a la vez, en torno a ella como hilo conductor, se gesta un proyecto donde se integran tres pilares básicos de las instituciones universitarias en pos de la formación integral de los estudiantes y de la resolución, al menos en parte, de problemas de la comunidad.

Los límites trazados entre docencia, extensión e investigación se vuelven difusos, generándose múltiples relaciones entre las diversas actividades, así como la participación de actores tanto universitarios como pertenecientes a instituciones de salud, en varias de estas instancias.

Un ejemplo de relación entre la docencia y la extensión se da porque los mismos estudiantes formados en el Módulo son los que luego se desempeñan como voluntarios para afianzar sus saberes en una actividad comunitaria. ¿Quién aprende en las instancias de promoción? ¿El voluntario o la madre destinataria? Ambos se enriquecen bajo una concepción dialógica del aprendizaje; saberes de libros y de la vida diaria se entrecruzan y funden. Los voluntarios recolectan datos mediante encuestas a las madres, analizan los resultados en encuentros con docentes y miembros de las instituciones para redefinir los ejes temáticos sobre los cuales enfatizar las acciones de promoción. Los estudiantes, mientras se forman, hacen actividades de extensión y también de investigación.

Algunos estudiantes voluntarios ingresaron como auxiliares estudiantiles de docencia a la cátedra de Bioquímica. Como tales colaboran en el desarrollo de los trabajos prácticos de la asignatura Bioquímica y también en el Módulo Optativo Lactancia Materna, donde asumen otro rol.
En cuanto a los docentes, ellos enseñan en el Módulo pero a la vez recolectan datos para servir de insumo a una investigación didáctica que se propone un análisis del dictado del Módulo. Los docentes también participan en docencia de posgrado. El contacto con profesionales de la salud facilita la detección de temas de importancia que se incorporan a los contenidos del Módulo. El elevado y creciente número de estudiantes interesados en cursar el Módulo llena de orgullo a los docentes pero a la vez genera un problema en cuanto al restringido espacio físico y a la inadecuada relación docente-alumno. Son muchos menos los alumnos interesados en el Voluntariado y menos aún los que participan en las actividades en el laboratorio de Bioquímica analizando muestras de leche materna. Recolectar las muestras implica el diálogo con la madre para interiorizarla de los objetivos de la investigación y del destino de la muestra que donará, previo consentimiento informado. Este momento también brinda una oportunidad de hablar de lactancia, de sus beneficios y de escuchar a las madres, deseosas de compartir sus inquietudes y sus satisfacciones. La investigación en el laboratorio también se entrelaza, en este caso, con la extensión.

Todo el proyecto en sus inicios estuvo a cargo de una única docente, quien concurrió a la capacitación que brindó la Asociación de Facultades de Ciencias Médicas de la República Argentina junto a Unicef. El desafío fue y continúa siendo comprometer a un mayor número de docentes y estudiantes en las actividades de docencia en el Módulo, extensión en el Voluntariado e Investigación en el PIUNT, generando un efecto multiplicador en estos tres ejes y asegurando la continuidad en el tiempo de las acciones.

Se podría concluir que en el marco de este proyecto los estudiantes fortalecen sus conocimientos disciplinares, desarrollan habilidades comunicacionales y aprenden de la comunidad. Las madres se fortalecen en sus decisiones, comparten saberes y dudas, y los niños se benefician de las bondades de la leche materna. La familia desarrolla sus vínculos con niños más sanos, no gasta dinero ni tiempo en preparar fórmulas; el beneficio se extiende a la sociedad en su conjunto.

Los voluntarios reconocen el valor de las actividades en la comunidad para su formación y también destacan la satisfacción personal que les brinda la acción solidaria. Las palabras que siguen, pertenecientes a Andrea Giorgis, una comprometida voluntaria durante varios años, reflejan el impacto de articular las actividades formativas con la extensión, entendida ésta no como una prolongación de la universidad sino como acciones de compromiso de una universidad inmersa en la sociedad que contribuye a su desarrollo y aprendiendo de ella:

"Me tomo hoy la atribución de revivir mi experiencia como voluntaria universitaria, y hablar en parte en mi nombre y en parte en el de otros, estudiantes y voluntarios (porque sabemos que lo seguiremos siendo si el crecimiento propio, en el saber, 
y el otro como persona, en el servicio, siguen siendo nuestras prioridades). Y quiero hablar también en su nombre porque compartieron este camino, porque cada uno aportó y cada uno recibió, y a pesar de las diferencias que, gracias a Dios, nos hacen únicos, creo que coincidirían conmigo si digo que no en vano, este camino que re-corremos se llama 'carrera universitaria'.

Porque corremos contra el tiempo, sorteamos obstáculos y vivimos tropiezos, nos sentimos a veces hábiles, a veces torpes, a veces juntos, a veces solos... y en esta senda donde el éxito y el fracaso depende de los ojos - y los principios- del espectador, se hace difícil no olvidar que: la meta es importante, pero más importante es quiénes somos al llegar a ella.

Y ahora, hablando por mí... en poco o en mucho, siento que no soy la misma gracias a éste, nuestro voluntariado, porque creo que representa en sí gran parte de lo que me llevo de esta carrera, como herramientas - y armas- para convertirme más que en la profesional, en la persona que quiero ser. Primero me llevo la experiencia de haber podido vivir lo que significa participar en todo el sentido, el gran sentido de la palabra, de la acción, de la vivencia, en su triple dimensión:

Participar formando parte, con sentido de pertenencia, no solo como grupo que camina y trabaja junto, sino como miembro de la comunidad a la que pertenezco.

Participar teniendo parte, asumiendo un rol, reconociendo la propia responsabilidad y desarrollando capacidades científicas y humanas.

Participar, en fin, tomando parte, influyendo a partir de la acción, y asumiendo el compromiso como propio. No hay mejor forma de invitar que desde adentro, no hay mejor forma de aprender que participando.

Me llevo el saber que en esta difícil transición que es la vida, donde lo natural es lo artificial, donde el ruido y la velocidad son moneda corriente, donde parece que llega más lejos quien camina solo, hay quienes se siguen animando a dar vida en un único y mágico momento donde todo cambia para siempre, y son capaces de afrontar el desafío de seguir dando - y recibiendo- vida a través de su pecho; y que una de las más valiosas y, curiosamente, menos costosas formas de involucrarnos en la tarea de mejorar nuestra salud, es trabajando para que estas madres sean muchas más. Finalmente me llevo la sensación de que esta carrera no fue fácil, pero a quien se le da mucho se le exige mucho, y yo tuve la oportunidad de ser, tener y tomar parte de esta experiencia, y con ello, de llevarme más de lo que dejé: lo aprendido, lo vivido y sobre todo la responsabilidad y el propósito de, en los caminos que me toque recorrer, seguir siendo voluntaria".

\section{6}

los voluntarios reconocen el valor de las actividades en la comunidad para su formación y también destacan la satisfacción personal que les brinda la acción solidaria.

\section{Agradecimientos}

Al Consejo de Investigaciones de la UNT y a la SPU por los subsidios, de investigación y de voluntariado recibidos.

\section{Referencias bibliográficas}

Edmond, K. M. et al (2006). Delayed Breastfeeding Initiation Increases Risk of Neonatal Mortality. Pediatrics, 117, 380-386.

Unicef (2012). Estado Mundial de la Infancia. Recuperado de: http://www.unicef. org/spanish/sowc2012/pdfs/SOWC\%202012\%20Main\%20Report\%20LoRes\%20 PDF_SP_03132012.pdf (21/06/2016).

Galli, A.; Pascual, L.; Rojo, H. y Ageitos M. L. (2009). Enseñanza de lactancia materna en las carreras de medicina: una experiencia de integración de conocimientos en un curso electivo. Revista Argentina de Educación Médica, 3, 30-39. Jones, G. et al (2003). How many child deaths can we prevent this year? Child Survival II. TheLancet, 362, 65-70.

Ministerio de Salud. Presidencia de la Nación Argentina (2007). Encuesta Nacional de Nutrición y Salud ENNYS. Recuperado de: http://www.msal.gob.ar/images/ stories/bes/graficos/0000000257cnt-a08-ennys-documento-de-resultados-2007. pdf (21/06/2016).

Ministerio de Salud de la Nación, Dirección Nacional de Maternidad e Infancia (2011). Situación de la LM en Argentina. Recuperado de: http://www.msal.gob.ar/ images/stories/bes/graficos/0000000215cnt-a11a-Situacion-de-la-lactancia-materna-2011.pdf (28/05/2016).

Waisman, M. (2003). Lactancia materna. Bases científicas para la práctica clínica. Módulo de autoinstrucción Recuperado de: http://www.unsa.edu.ar/bibsalud/descargas/Lactancia_Materna.pdf (22/06/2016). 